\title{
Update of the Recommendations for the Use of Once-Weekly Isoniazid-Rifapentine Regimen to Latent Mycobaterium tuberculosis Infection*
}

\author{
*Borisov AS, Bamrah Morris S, Njie GJ, Winston CA, Burton D, Goldberg S, et al. \\ Update of recommendations for use of once-weekly isoniazid-rifapentine \\ regimen to treat latent Mycobacterium tuberculosis infection. \\ MMWR Morb Mortal Wkly Rep 2018;67:723-6.
}

Derya Alabaz ${ }^{1}$

${ }^{1}$ Department of Pediatric Infectious Diseases, Cukurova University School of Medicine, Adana, Turkey

Update of the Recommendations for the Use of Once-Weekly Isoniazid-Rifapentine Regimen to Latent Mycobaterium Tuberculosis Infection. Morbidity and Mortality Weekly Report (MMWR)-June 29, 2018/67(25);723-726 CDC (Centers for Disease Control and Prevention)

Treatment of latent tuberculosis infection (LTBI) has a critical importance in the control and elimination of tuberculosis (TB) in the United States of America. In 2011, the Centers for Disease Control and Prevention (CDC) recommended the use of a directly observed therapy (DOT) regimen (HP) including the once-weekly use of isoniazid-rifapentine in a period of 12 weeks in pediatric patients aged $\geq 12$ years and in patients with HIV (1).

CDC addressed the updated recommendations (by including self-administered therapy (SAT) to the $3 \mathrm{HP}$ regimen) for the $3 \mathrm{HP}$ treatment in this population. A CDC Workgroup carried out a systematic evaluation investigating the meta-analyses of 3HP use adapted to the Guide to Community Preventive
Services in 2017. Nineteen articles representing a total of 15 different studies were included, and it was indicated that the $3 \mathrm{HP}$ regimen was as safe and effective as other $\mathrm{LTBI}$ regimens and that a higher level of treatment completion was reached.

The Workgroup presented the results of the meta-analysis to a group of TB experts in July 2017, and CDC solicited input from the Advisory Council for the Elimination of Tuberculosis (ACET) and from the members of the public in December 2017.

While CDC maintained its recommendations to use the $3 \mathrm{HP}$ regimen in the treatment of $\mathrm{LTBI}$ in adults, it also included the following to the use of the $3 \mathrm{HP}$ regimen:

1. In persons with LTBI aged 2-17 years

2. In persons with LTBI, who are infected with HIV (including the Acquired Immune Deficiency Syndrome (AIDS)) and who receive antiretroviral medication that have acceptable drug-drug interactions with rifapentin.

3. In children aged $\geq 2$ years by DOT or SAT.

Correspondence Address / Yazışma Adresi

Derya Alabaz

Çukurova Üniversitesi Tıp Fakültesi

Çocuk Enfeksiyon Hastalıkları Bilim Dalı,

Adana-Türkiye

E-mail: deryaalabaz@yahoo.com 


\section{Systematic Review}

The Workgroup of the CDC, including epidemiologists, health scientists, physicians from CDC's Turberculosis Elimination Program and a CDC library specialist, came together in order to conduct a literature review using the methods adapted from the Guide to Community Preventive Services. The library specialist carried out a systematic research on studies regarding $3 \mathrm{HP}$ used for the treatment of LTBI on databases such as MEDLINE, Embase, CINAHL, Cochrane Library, Scopus and Clinicaltrials. gov. So as to identify the studies that could be overlooked, the reference list of the articles included were reviewed, and the TB experts of the CDC were counselled. The collected works included English articles meeting the following criteria:

1. The study design was randomized-control, experimental, quasi-experimental, observational, observation cohort or concurrent comparison group studies.

2. Target groups including (but not limited to) patients aged $\geq 12$ years, children aged 2-11 years, and persons infected with HIV.

3. The recorded data: prevention rate of TB related to the $3 \mathrm{HP}$ regimen, the rate of treatment completion, side effects, treatment discontinuation due to side effects or death.

Two separate individuals from the CDC Workgroup independently scanned the obtained citations and retrieved fulltext articles in the related literature. Using a standard data abstraction form, the reviewers summarized the data in relation to acceptance qualities, outcomes of interest, demographics, benefits, harms, points to consider during treatment, and evidence gaps. Each and every study was assessed according to the inclusion and exclusion criteria of the Guide to Community Preventive Services $(2,3)$. The unconformity between the reviewed studies were solved with the consensus reached by the members of the CDC Workgroup.

The CDC Workgroup evaluated thirty full-text articles out of 292 citations. No eligible studies including children aged < 2 years were found. A total of 19 articles representing 15 valuable studies were included into the meta-analysis. The meta-analysis showed that $3 \mathrm{HP}$ was as safe and effective as other LTBI regimens and that a significantly higher level of treatment completion was reached (4). The majority of the studies included had, in general, a good design and good implementation quality. The most frequent limitations were inadequate reporting of suitable analytic methods and probable selection bias.

Randomized-control studies and drug-interaction studies found predominantly in the meta-analyses published recently were summarized as follows (5-9):

\section{HP Study in Children}

In a large, randomized clinical study where 3HP was administered by DOT, it was shown that children aged 2-17 years demonstrated fair tolerance and that it was as effective as 9-month daily isoniazid regimen (9H) to prevent TB (5). Moreover, the study also put forth that 3HP was safe and a higher rate of treatment completion was achieved compared to the $9 \mathrm{H}$ regimen (5). Present data on the safety and pharmacokinetics of rifapentine in children aged $<2$ years was not found.

\section{HP study in HIV-infected patients, including AIDS}

In 2011, CDC recommended the use of 3HP regimen in the treatment of LTBI in healthy individuals infected with HIV (including those with AIDS) who do not receive antiretroviral drugs (1). Since then, additional data confirm that $3 \mathrm{HP}$ regimen is not only affective in persons infected with HIV but also demonstrate the absence of a clinically significant drug interaction between rifapentine (once a week) and efavirenz/ raltegavir (4,6-8).

\section{Study of Self-Administered (SAT) Treatment}

When a randomized clinical study including persons aged $\geq 18$ years in the USA compared the 3HP-DOT and 3HP-SAT in terms of treatment completion and safety, primary evidence in support of the 3HP-SAT treatment was found (9). Randomized-control studies evaluating patients under the age of 18 for 3HP-SAT treatment were not found.

\section{Expert Consultation}

In July 2017, CDC came together with nine experts (not from $(D C$ ) for the diagnosis, treatment, prevention, observation, epidemiology, clinical research, pulmonology, pediatrics of TB and LTBI, HIV/AIDS, public health programs and patient rights. The systematically reviewed results and recommendations of the CDC were presented to the experts

1. With individual viewpoints regarding the review,

2. Experienced in the implementation of the $3 \mathrm{HP}$ regime in various settings and populations,

3. With individual viewpoints regarding the proposed updates.

Experts who had implemented 3HP in their patients presented the benefits of the regimen including its rising acceptance and the completion of the treatment. Some experts reported that the $3 \mathrm{HP}$ regimen had been used with high treatment adherence in children as small as 2 years of age in many health departments. Some indicated in 2011 that the recommendation of $3 \mathrm{HP}$ regimen with DOT would cause restrictions in usage. CDC requested the final recommendations of the public and ACET in December 2017. 
The 2011 recommendations included limited use of the $3 \mathrm{HP}$ regimen in children aged $\leq 12$ years in the treatment of LTBI in pediatrics (1). New data related to the efficiency and safety of $3 \mathrm{HP}$ in children were found sufficient to be used in the treatment of $L T B I$ in children aged $\geq 2$ years (4).

In HIV-infected patients, the information on the interaction of rifamycin containing agents (i.e. rifampin, rifabutin and ifapentin) among specific antimicrobial agents and antiretroviral agents is found in the "HIV-1 Infected Adults and Adolescents Section" of the guidelines of the U.S. Department of Health and Human Services. In this frequently updated guideline, there is a section dealing with LTBI treatment in HIV-infected patients and tables regarding drug interactions*. Use of concomitant LTBI treatment and antiretroviral agents should be carried out by clinicians experienced in the management of both conditions.

In 2011, CDC recommended the application the 3HP regimen by DOT (1). The rate of treatment completion rose to its highest level by DOT; however, it was seen that the responsibility and cost of the DOT were higher than SAT (9). During expert consultation and ACET review, some experts strongly recommended the permission to be given to the use of SAT in children aged $\geq 2$ years under clinical monitoring. Based on this expert view, ACET officially recommended the expansion of parentally administered SAT in children. These experts still opt for the administration of DOT in children aged 2-5 years in the treatment of LTBI, justifying that TB advancement and the risk of severe disease are much higher in children when compared to older children and adults. Healthcare providers should reach to a mutual understanding with each and every patient (along with his/her parent or legal guardian) for the administration of the SAT program (by taking into account the sources and age of the patient, medical history, social conditions and risk factors leading to severe TB). Subject matter experts emphasized the importance of drug suppliers for $3 \mathrm{HP}$ and patient education.

\section{Recommendations}

While $\mathrm{CDC}$ maintains the recommendation of the $3 \mathrm{HP}$ regimen for the treatment of LTBI in adults taking into consideration the systematic reviews on efficacy, safety and rates of treatment completion and the viewpoint of tuberculosis experts and ACET and the public, new recommendations for the use of the 3HP regimen are as follows:

1. In persons with $L T B I$ aged 2-17 years,

2. In persons with HIV (including AIDS) and those receiving antiretroviral drugs that have acceptable drug -druginteraction with rifapentin HIV,

3. In children aged $\geq 2$ years

should opt for SAT or DOT administration.
The healthcare provider should choose the method of administration as DOT vs SAT (depending on local practice, individual patient qualities and preferences, and other matters including the risk of advancement to severe tuberculosis diseases). Concurrent LTBI treatment and the use of antiretroviral agents should be directed by clinicians experienced in the management of both cases (Box 1).

Box 1. Updated Recommendations for the Use of Once-Weekly Isoniazid-rifapentine Regimen for 12 weeks for the Treatment of Latent Mycobaterium tuberculosis Infection

CDC maintains recommending the use of the short-course combination regimen of once-weekly isoniazid-rifapentine for 12 weeks (3HP) for the treatment of latent tuberculosis infection (LTBI) in adults. With regard to age limits, HIV infection, and administration of the treatment, CDC now also recommends the following:

- $\quad$ Use of 3HP in persons aged 2-17 years;

- Use of $3 \mathrm{HP}$ in persons with LTBI who are living with human immunodeficiency virus (HIV) infection, including acquired immunodeficiency syndrome (AIDS) and taking antiretroviral medications with acceptable drug-drug interactions with rifapentine*; and

- $\quad$ Use of 3HP by directly observed therapy (DOT) or self-administered therapy (SAT) in persons aged $\geq 2$ years; the health care provider should choose the mode of administration (DOT versus SAT) based on local practice, individual patient attributes and preferences, and other considerations, including risk for progression to severe forms of tuberculosis disease.

*https://aidsinfo.nih.gov/guidelines/html/1/adult-and-adolescentarv/367/overview.

Box 2. Guidance to health care providers during treatment of latent tuberculosis infection (LTBI) with a combination regimen of isoniazid and rifapentine in 12 once-weekly doses (3HP)

- $\quad$ Evaluate all patients for active tuberculosis disease both before and during treatment of LTBI.

- $\quad$ Inform the patient or parents or legal guardians about possible adverse effects and instruct them to seek medical attention when symptoms of possible adverse reaction first appear; particularly drug hypersensitivity reactions, rash, hypotension, or thrombocytopenia.

- Conduct monthly evaluations to assess treatment adherence and adverse effects, with repeated patient education regarding adverse effects at each visit.

- Order baseline hepatic chemistry blood tests (at least aspartate aminotransferase [AST]) for patients with the following specific conditions: human immunodeficiency virus infection, liver disorders, postpartum period ( $\leq 3$ months after delivery), regular alcohol use, injection drug use, or use of medications with known possible interactions.

- Conduct blood tests at subsequent clinical encounters for patients whose baseline testing is abnormal and for others at risk for liver disease. Discontinue $3 \mathrm{HP}$ if a serum AST concentration is $\geq 5$ times the upper limit of normal in the absence of symptoms or $\geq 3$ times the upper limit of normal in the presence of symptoms.

- In case of a possible severe adverse reaction, discontinue 3HP and provide supportive medical care. Conservative management and continuation of $3 \mathrm{HP}$ under observation can be considered in the presence of mild to moderate adverse events as determined by health care provider. 


\section{Patient Monitoring and Side Effects}

Prior to 3HP treatment, hepatic enzyme and other blood tests should be performed for some patients (Box 2). Approximately $4 \%$ of the patients using 3HP show flu-like symptoms such as fever, headache, dizziness, nausea, muscle and bone pain, rash, itchiness, and red eye or symptoms like other systemic drug reactions $(4,10)$. Nearly $5 \%$ of the people quit the $3 \mathrm{HP}$ treatment due to side effects as systemic drug reactions, which typically reveal after the third or fourth dosage and start about four hours after drug intake $(4,10)$. Hypotension and syncope have been rarely reported (in two of every 1000 patients treated) $(4,10)$. 3HP treatment should be discontinued while the cause is being identified if symptoms suggest a systemic drug reaction. The symptoms usually improve without treatment in 24 hours. Neutropenia and an increase in liver enzymes are rarely encountered $(4,10)$. CDC recommends the healthcare provider to guide the patients in informing the side effects. Should patients use symptom control lists, timely identification and reporting may become easier ${ }^{\dagger}$.

Rifapentine, a compound or rifamycin, induces the metabolism of many drugs like rifampin. CDC recommends the monitoring of the patients when drugs that interact with $3 \mathrm{HP}$ (i.e. methadone or warfarin) are prescribed. Rifapentine can decrease the efficiency of hormonal contraceptives, and thus, women using hormonal birth control should be advised to use a barrier method or simply to change their birth control method. Women should be advised to inform the healthcare provider in the event of a pregnancy or should they decide to try to become pregnant.

Patients in 3HP SAT should be encouraged to record their drug intakes and report any diversions from the anticipated regimen since a change in doses can reduce efficiency or safety. Individuals given $3 \mathrm{HP}$ regimen should be monthly assessed in person or by phone in terms of compliance and side effects.

Further studies are needed in order to understand the pharmacokinetics, safety and tolerance of $3 \mathrm{HP}$ in children aged $<2$ years, adherence and safety of the 3HP-SAT treatment in patients aged $<18$ years, and safety of $3 \mathrm{HP}$ in pregnant patients (4).

Any side effect related to the LTBI treatment leading to hospital admittance or morbidity should be reported to local or state health departments by the healthcare professionals for their inclusion in the 'National Surveillance for Severe Adverse Events Associated with Treatment for LTBI (e-mail: Itbidrugevents@cdc.gov).

Serious drug side effects, product quality problems, and therapeutic failures should be reported to the Food and Drug Administration's MedWatch program (https://www.fda.gov/ Safety/MedWatch/HowToReport/default.htm) or by telephoning 1-800-FDA-1088.
Additional information regarding $3 \mathrm{HP}$ is available at https://www.cdc.gov/tb/publications/ltbi/ltbiresources.htm. Questions can also be directed to CDC's Division of Tuberculosis Elimination by e-mail (vog.cdc@ofnicdc) or by telephoning 800-CDC-INFO (800-232-4636).

*https://aidsinfo.nih.gov/guidelines/html/1/adult-and-adolescent-arv/367/overview.

'Examples of patient's medication intake log and symptoms checklists are available at https://www.cdc.gov/tb/publications/pamphlets/12-doseregimen.htm.

\section{References}

1. $C D C$. Recommendations for use of an isoniazid-rifapentine regimen with direct observation to treat latent Mycobacterium tuberculosis infection. MMWR Morb Mortal Wkly Rep 2011;60:1650-3.

2. Briss PA, Zaza S, Pappaioanou M, Wright-De Agüero L, Truman Bl, Hopkins DP, et al; The Task Force on Community Preventive Services. Developing an evidence-based Guide to Community Preventive Servicesmethods. Am J Prev Med 2000;18(Suppl):S35-43.

3. Zaza S, Wright-De Agüero LK, Briss PA, Truman BI, Hopkins DP, Hennessy $\mathrm{MH}$, et al; Task Force on Community Preventive Services. Data collection instrument and procedure for systematic reviews in the Guide to Community Preventive Services. Am J Prev Med 2000;18(Suppl):44-7.

4. Njie GJ, Morris SB, Woodruff RY, Moro RN, Vernon AA, Borisov AS. Isoniazid-rifapentine for latent tuberculosis infection: a systematic review and meta-analysis. Am J Prev Med 2018. Epub June 11, 2018.

5. Villarino ME, Scott NA, Weis SE, Weiner M, Conde MB, Jones B, et al; International Maternal Pediatric and Adolescents AIDS Clinical Trials Group; Tuberculosis Trials Consortium. Treatment for preventing tuberculosis in children and adolescents: a randomized clinical trial of a 3-month, 12-dose regimen of a combination of rifapentine and isoniazid. JAMA Pediatr 2015;169:247-55.

6. Sterling TR, Scott NA, Miro JM, Calvet G, La Rosa A, Infante R, et al; Tuberculosis Trials Consortium; AIDS Clinical Trials Group for the PREVENT TB Trial. Three months of weekly rifapentine and isoniazid for treatment of Mycobacterium tuberculosis infection in HIV-coinfected persons. AIDS 2016;30:1607-15.

7. Weiner M, Egelund EF, Engle M, Kiser M, Prihoda TJ, Gelfond JA, et al. Pharmacokinetic interaction of rifapentine and raltegravir in healthy volunteers. J Antimicrob Chemother 2014;69:1079-85.

8. Podany AT, Bao Y, Swindells S, Chaisson RE, Andersen JW, Mwelase T, et al; AIDS Clinical Trials Group A5279 Study Team. Efavirenz pharmacokinetics and pharmacodynamics in HIV-infected persons receiving rifapentine and isoniazid for tuberculosis prevention. Clin Infect Dis 2015;61:1322-7.

9. Belknap R, Holland D, Feng PJ, Millet JP, Caylà JA, Martinson NA, et al; TB Trials Consortium iAdhere Study Team. Self-administered versus directly observed once-weekly isoniazid and rifapentine treatment of latent tuberculosis infection: a randomized trial. Ann Intern Med 2017;167:689-97.

10. Sterling TR, Moro RN, Borisov AS, Phillips E, Shepherd G, Adkinson NF, et al; Tuberculosis Trials Consortium. Flu-like and other systemic drug reactions among persons receiving weekly rifapentine plus isoniazid or daily isoniazid for treatment of latent tuberculosis infection in the PREVENT Tuberculosis study. Clin Infect Dis 2015;61:527-35. 